\title{
Latest results from the MINOS experiment
}

\author{
Justin EVANS*䄱 \\ University College London \\ E-mail: evansj@hep.ucl.ac.uk
}

\begin{abstract}
The MINOS experiment utilizes the NuMI neutrino beam to study the phenomenon of neutrino oscillations. Muon neutrinos are sent over a baseline of $735 \mathrm{~km}$, with a detector near the production point at Fermilab and one at the Soudan underground laboratory in northern Minnesota. By observing the $v_{\mu}$ disappearance characteristic of oscillations, MINOS can measure the oscillation parameters. MINOS has previously made the best measurement of the atmospheric-regime mass splitting to date. New results are presented in which the data-set is doubled. Further analysis improvements, and the inclusion of additional event samples, further improve the sensitivity to the oscillation parameters. The mixing angle $\theta_{13}$ is currently not measured to differ from zero. By searching for $v_{e}$ appearance in the $v_{\mu}$ beam, MINOS is able to set new limits on the value of $\theta_{13}$. An observation of the neutral current interaction rate at the far detector allows limits to be placed on the existence of sterile neutrinos. From September 2009 to March 2010, MINOS has taken data with a dedicated $\bar{v}_{\mu}$ beam, allowing the first direct precision measurement of the antineutrino oscillation parameters in the atmospheric regime.
\end{abstract}

35th International Conference of High Energy Physics

July 22-28, 2010

Paris, France

\footnotetext{
* Speaker.

${ }^{\dagger}$ For the MINOS collaboration.
} 


\section{The MINOS experiment}

The MINOS neutrino oscillation experiment uses the NuMI facility to produce a $v_{\mu}$ or $\bar{v}_{\mu}$ beam. Two magnetized steel-scintillator calorimeters [1], the near detector (ND) at Fermilab, and the far detector (FD) $735 \mathrm{~km}$ from the neutrino source, measure the neutrino energy spectra before and after oscillations have occurred. This two-detector arrangement makes oscillation measurements robust against many sources of systematic uncertainty which affect both detectors similarly.

\section{Muon neutrino disappearance search}

The neutrino oscillations measured by MINOS are driven by the largest neutrino mass splitting $\Delta m_{\mathrm{atm}}^{2}$. The primary oscillation signal observed by MINOS is the disappearance of muon neutrinos. The magnitude of the disappearance is governed by the mixing angle $\theta_{23}$. Observation of this energy-dependent disappearance allows a measurement of $\Delta m_{\text {atm }}^{2}$ and $\sin ^{2}\left(2 \theta_{23}\right)$. The charged current (CC) interactions of muon neutrinos, $v_{\mu}+N \rightarrow \mu^{-}+N^{\prime}$ (where $N$ is a nucleus in the detector), are used for this analysis since the muon produced tags the flavour of the incoming neutrino.

The result reported here updates the previously published result [2]. The data-set is more than doubled. Additionally, a number of analysis improvements further increase the sensitivity to the oscillation parameters. An improved method of identifying CC $-v_{\mu}$ interactions improves the selection efficiency, particularly at low energies [3]. Cuts are no longer applied on the charge of the reconstructed muon, regaining mis-identified CC- $v_{\mu}$ events at low energies [4]. A new hadronic shower energy estimator improves the hadronic energy resolution by more than $30 \%$ at low energies [5]. Events are the FD are separated into bins according to their energy resolution, maximizing the use of oscillation information contained in the most well-measured events [6].

At the FD, $2451 \mathrm{CC}-v_{\mu}$-like events are observed under the assumption of no oscillations. 1986 events are observed. The observed and expected FD energy spectra are shown in figure 1. The observed energy spectrum is fit for oscillations, including the dominant sources of systematic uncertainty as penalty terms in the fit. This fit yields a measurement of the oscillation parameters of $\Delta m_{\mathrm{atm}}^{2}=2.35_{-0.08}^{+0.11} \times 10^{-3} \mathrm{eV}^{2}, \sin ^{2}\left(2 \theta_{23}\right)>0.91$ (90\% C.L.). The allowed regions for the neutrino oscillation parameters are shown in figure 2. The energy dependence of the observed deficit at the FD allows the process behind the disappearance to be investigated. Pure neutrino decay [8] is disfavoured at better than $6 \sigma$; pure neutrino decoherence [9] is disfavoured at better than $8 \sigma$.

\section{Electron neutrino disappearance search}

The third neutrino mass state is predominantly an almost equal mix of $v_{\mu}$ and $v_{\tau}$ flavour. The possibility remains of a small $v_{e}$ component, the size of which is parameterized by the angle $\theta_{13}$; this angle has not been measured to differ from zero. A non-zero value of $\theta_{13}$ would result in $v_{e}$ appearance in the $v_{\mu}$ beam at the MINOS FD.

Electron neutrinos are tagged by their CC interactions $v_{e}+N \rightarrow e^{-}+N^{\prime}$. The electron produces an electromagnetic shower: a much denser energy deposition than the hadronic shower of typical NC interactions. To select CC- $v_{e}$ events, eleven variables are used which characterize the shape of the energy deposition in the shower. These variables are combined in a neural network to produce a single selection variable. 


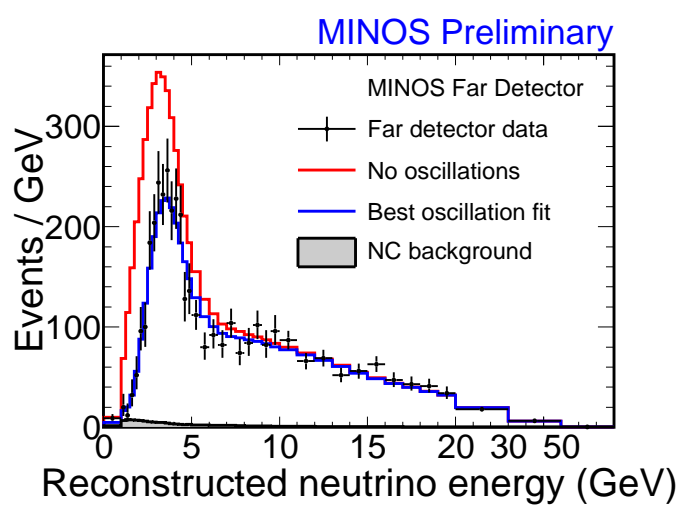

Figure 1: The reconstructed energy spectrum of selected charged current $v_{\mu}$ events at the far detector, also showing the prediction in the case of no disappearance and the best fit to oscillations.

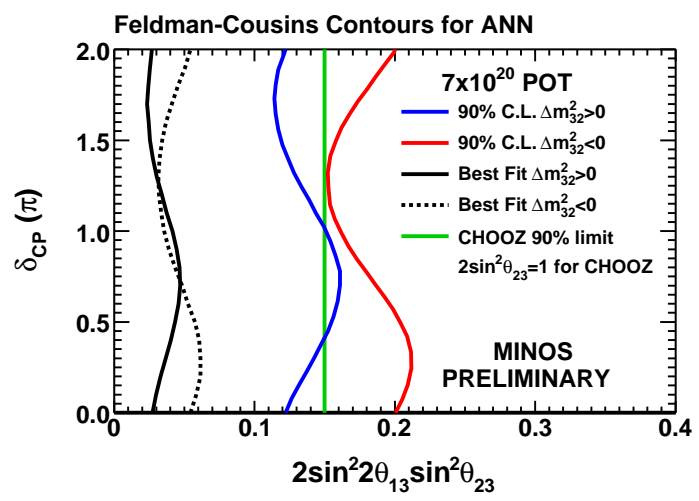

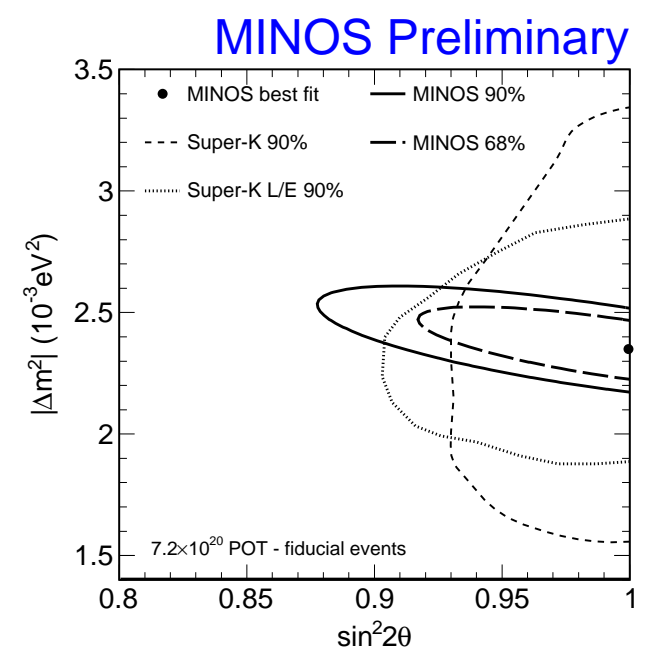

Figure 2: Regions of oscillation parameter space allowed by the MINOS data, compared to other experimental results [7].

Figure 3: The MINOS $90 \%$ allowed regions for $2 \sin ^{2}\left(2 \theta_{13}\right) \sin ^{2}\left(\theta_{23}\right)$ for the normal (blue) and inverted (red) mass hierarchies. Also shown are the MINOS best fit value (black) and the Chooz $90 \%$ limit [11] (green).

At the FD, $49.1 \pm 7.0$ (stat.) \pm 2.7 (syst.) background events are expected. $54 v_{e}$-like events are observed. This $0.7 \sigma$ excess shows no evidence for $v_{e}$ appearance. This observation can be interpreted as a limit on the size of $\theta_{13}$. The limit depends on the size of the unknown parameter $\delta_{\mathrm{CP}}$ (which describes the amount of $\mathrm{CP}$ violation in the neutrino sector), and the mass hierarchy (the sign of $\Delta m_{\mathrm{atm}}^{2}$ ), and is shown in figure 3 [10].

\section{Neutral current disappearance search}

The NC interaction rates of all three active neutrino flavours are identical. Therefore oscillations between the three active flavours produce no depletion in the NC event rate. Any deficit in the $\mathrm{NC}$ event rate would thus be evidence for oscillation to one or more sterile neutrino flavours.

$\mathrm{NC}$ events consist of hadronic showers with no associated muon. The selection criteria look for these shower-like events. At the FD, 757 events are expected. 802 NC-like events are observed, 


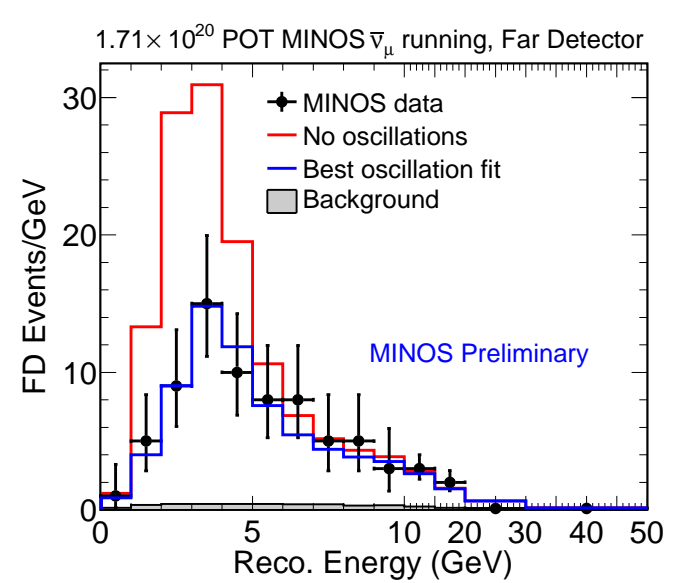

Figure 4: The reconstructed energy spectrum of selected CC- $\bar{v}_{\mu}$ events at the FD. Also shown are the prediction in the case of no disappearance and the best fit of this data to oscillations.

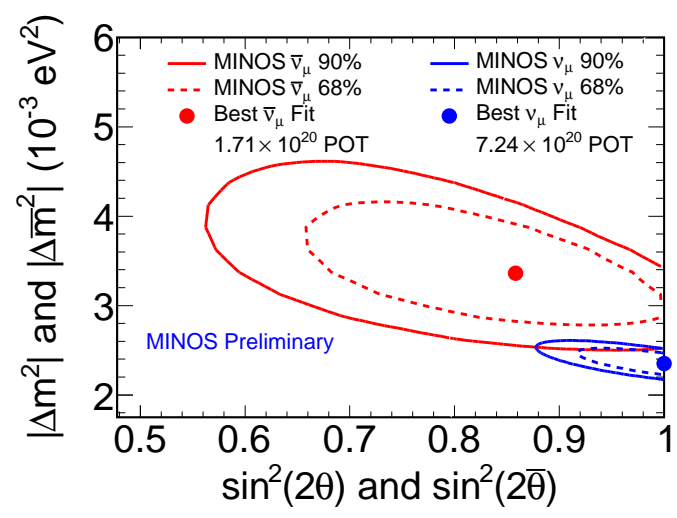

Figure 5: Regions of $\bar{v}_{\mu}$ oscillation parameter space allowed by the MINOS data, compared to the MINOS $v_{\mu}$ measurement.

showing no evidence for oscillation into sterile neutrinos.

Of the muon neutrinos known to have disappeared from the $\mathrm{CC}-v_{\mu}$ measurement, the fraction which have oscillated to sterile neutrinos can be limited to less than 0.22 (90\% C.L.), assuming no $v_{e}$ appearance $\left(0.40\right.$ for $\left.\theta_{13}=11.5^{\circ}\right)$.

\section{Muon antineutrino disappearance search}

The NuMI beam can be configured to produce a $\bar{v}_{\mu}$-enhanced beam. By observing the charge of the muon produced, MINOS is able to perform excellent event-by-event separation of CC $v_{\mu}$ and $\bar{v}_{\mu}$ interactions, achieving a CC- $\bar{v}_{\mu}$ purity of $98 \%$ below $6 \mathrm{GeV}$.

At the FD, $155 \mathrm{CC}-\bar{v}_{\mu}$ events are expected without oscillations; 97 are observed. Figure 4 shows the predicted and observed energy spectra. A fit to oscillations yields a best value for the antineutrino oscillation parameters of $\Delta \bar{m}_{\mathrm{atm}}^{2}=3.36_{-0.40}^{+0.45} \times 10^{-3} \mathrm{eV}^{2}, \sin ^{2}\left(2 \bar{\theta}_{23}\right)=0.86 \pm 0.11$.

The allowed region for the antineutrino oscillation parameters is shown in figure 5, compared to the MINOS allowed region measured with $v_{\mu}$ interactions. A tension exists between the MINOS $v_{\mu}$ and $\bar{v}_{\mu}$ measurements which is being addressed with additional $\bar{v}_{\mu}$ data taking.

\section{References}

[1] Nucl. Instrum. and Meth. A 596, 190 (2008).

[2] Phys. Rev. Lett. 101, 131802 (2008).

[3] J. Ratchford, Ph.D. Thesis, U-T Austin (2011).

[4] S. Coleman, Ph.D. Thesis, C. William \& Mary (2010).

[5] C. Backhouse, Ph.D. Thesis, U. Oxford (2011).

[6] J. Mitchell, Ph.D. Thesis U. Cambridge (2011).
[7] Phys. Rev. Lett. 93, 101801 (2004); Phys. Rev. D 71, 112005 (2005).

[8] Phys. Rev. Lett, 82, 2640 (1999).

[9] Phys. Rev. D 67, 093006 (2003).

[10] Phys. Rev. D 82, 051102 (2010).

[11] Eur. Phys. J. C 22, 221 (2003). 